\title{
Clinical and surgical management of a congenital Type II split cord malformation presenting with progressive cranial neuropathies: case report
}

\author{
Patrick R. Maloney, MD, ${ }^{1}$ Meghan E. Murphy, MD, ${ }^{1}$ Molly J. Sullan, MS, ${ }^{2}$ Kathryn M. Van Abel, MD, ${ }^{2}$ \\ Shelagh A. Cofer, MD, ${ }^{2}$ John C. Cheville, MD, ${ }^{3}$ and Nicholas M. Wetjen, MD' \\ Departments of ${ }^{1}$ Neurosurgery, ${ }^{2}$ Otolaryngology, and ${ }^{3}$ Pathology, Mayo Clinic School of Medicine, Rochester, Minnesota
}

Split cord malformation (SCM) is a rare abnormality of notochord development. The majority of cases occur in the thoracolumbar region, with more than 30 cases of cervical SCM reported. The clinical impact of SCMs involving the cervical cord is therefore largely unknown. In addition, the concomitant finding of brainstem involvement is presumably incompatible with life in the majority of patients, resulting in a paucity of data regarding this clinical scenario. In this paper the authors present the first case, to their knowledge, of an incomplete cervical SCM involving the brainstem and discuss its clinical impact, diagnosis, and management.

https://thejns.org/doi/abs/10.3171/2016.9.PEDS15661

KEY WORDS split cord malformation; duplicated spinal cord; diplomyelia; brainstem hypoplasia; cervical cord malformation; unilateral vocal cord palsy; spine

$\mathrm{S}$ PLIT cord malformation (SCM) is a rare abnormality of notochord development. The majority of cases occur in the thoracolumbar region, ${ }^{12}$ with more than 30 cases of cervical SCM reported. ${ }^{2}$ The clinical impact of SCMs involving the cervical cord is therefore largely unknown. In addition, the concomitant finding of brainstem involvement is presumably incompatible with life in the majority of patients, resulting in a paucity of data regarding this clinical scenario. In this report we present the first case, to our knowledge, of an incomplete cervical SCM involving the brainstem, and discuss its clinical impact, diagnosis, and management.

\section{Case Report}

History and Examination

The patient was born via induced vaginal delivery at 38 weeks due to maternal hypertension, with no perinatal complications. Her parents noted apneic episodes, progressive lethargy, difficulty feeding, and noisy breathing during sleep. On Day 7 of life, she became cyanotic and required cardiopulmonary resuscitation by a family member; emergency medical services was activated and she was initially evaluated at the local hospital. She was subsequently transferred to our tertiary care center after a series of medically witnessed apneic episodes associated with biphasic stridor.

On arrival, she was hemodynamically stable on continuous positive pressure support. She had no obvious dysmorphic features. An isolated capillary hemangioma was noted on her arm, and she was observed to have unilateral left-sided torticollis. Flexible endoscopy revealed a left vocal cord paresis, which was confirmed during flexible sleep-state endoscopy. She had an otherwise normal airway examination. An immature suck-swallow-breath sequence was diagnosed via functional endoscopic examination of swallowing (FEES). Aspiration with thin liquids was noted on video fluoroscopic swallowing examination and both central apneas and hypopneas were noted on polysomnography.

Magnetic resonance imagining of the brain and spine revealed a 6-mm-long Type II cervical SCM (Figs. 1 and 2 ), with no evidence of syringohydromyelia or cord compression. Also notable was hypoplasia of the left dorsolateral medulla. A chromosomal microarray was normal, and workup for infection was negative. There was no evidence of spinal cord tethering and the patient demonstrated clinical improvement with successful advancement of oral feedings and stable oxygen saturations with decreased apnea, therefore conservative neurosurgical management

ABBREVIATIONS AHI = apnea-hypopnea index; BAER = brainstem auditory evoked response; FEES = functional endoscopic examination of swallowing; SCM = split cord malformation; TCS $=$ tethered cord syndrome.

SUBMITTED February 11, 2016. ACCEPTED September 14, 2016.

INCLUDE WHEN CITING Published online December 23, 2016; DOI: 10.3171/2016.9.PEDS15661. 


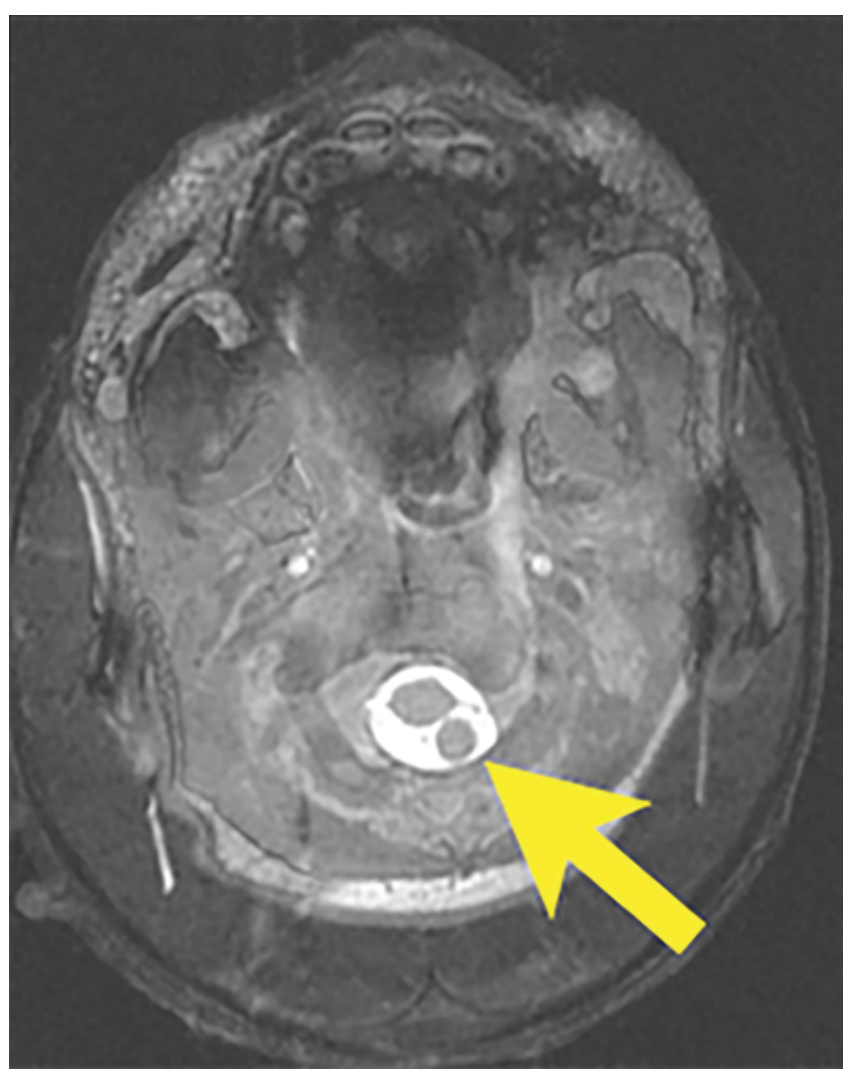

FIG. 1. Preoperative axial contrast-enhanced MR image showing the SCM (arrow). Figure is available in color online only.

was recommended. After demonstrating weight gain, an improved suck-swallow-breath sequence, and fewer apneic events over the subsequent 2 weeks, she was discharged on room air with overnight oximetry.

At the 6-week follow-up evaluation, she was found to have decreased aspiration on video swallow, but persistent laryngeal penetration on FEES. Her unilateral vocal cord paresis and torticollis were unchanged, but her parents reported decreased apneic spells and noisy breathing.

At her 6-month evaluation, she was noted to have progressive lower cranial nerve dysfunction (cranial nerves XII, XI, X, and IX), right-sided motor preference versus subtle left-sided hemiparesis, and worsening torticollis with tightening of the left sternocleidomastoid and erector spinae muscles. Swallow studies were repeated and found to be negative for aspiration, with good coordination of her suck-swallow-breathe reflex. However, due to persistent concerns for aspiration, microdirect laryngoscopy, bronchoscopy with bronchoalveolar lavage, and upper endoscopy with biopsy were performed to definitively assess for microaspiration. She was found to have an abnormal 24-hour intraesophageal pH study with elevated BoixOchoa score and her airway pepsin study was negative. ${ }^{3}$

FEES revealed mild pharyngeal weakness, suggested by retention of formula in both pyriform sinuses and some collection along the postcricoid space. Repeat polysomnography revealed an apnea-hypopnea index (AHI) score of 46 without supplemental oxygen and 3 with low-flow oxygen at $0.25 \mathrm{~L}$. Nocturnal oxygen supplementation with continued oximetry was initiated. Brainstem auditory evoked responses (BAERs) showed all waveforms present; however, waveforms on the left were better defined than those on the right. Somatosensory evoked potentials showed no obvious impaired conduction, but there are no well-established normal values in this young age group that allow for detection of mild slowing. Given that central and peripheral myelination may not be complete, changes in waveforms and latencies may not be comparable to normal adult values. ${ }^{7}$ Computed tomography of the cervical spine revealed a hypoplastic left C-1 lateral mass, neural arch, and left occipital condyle compared with the right (Fig. 3).

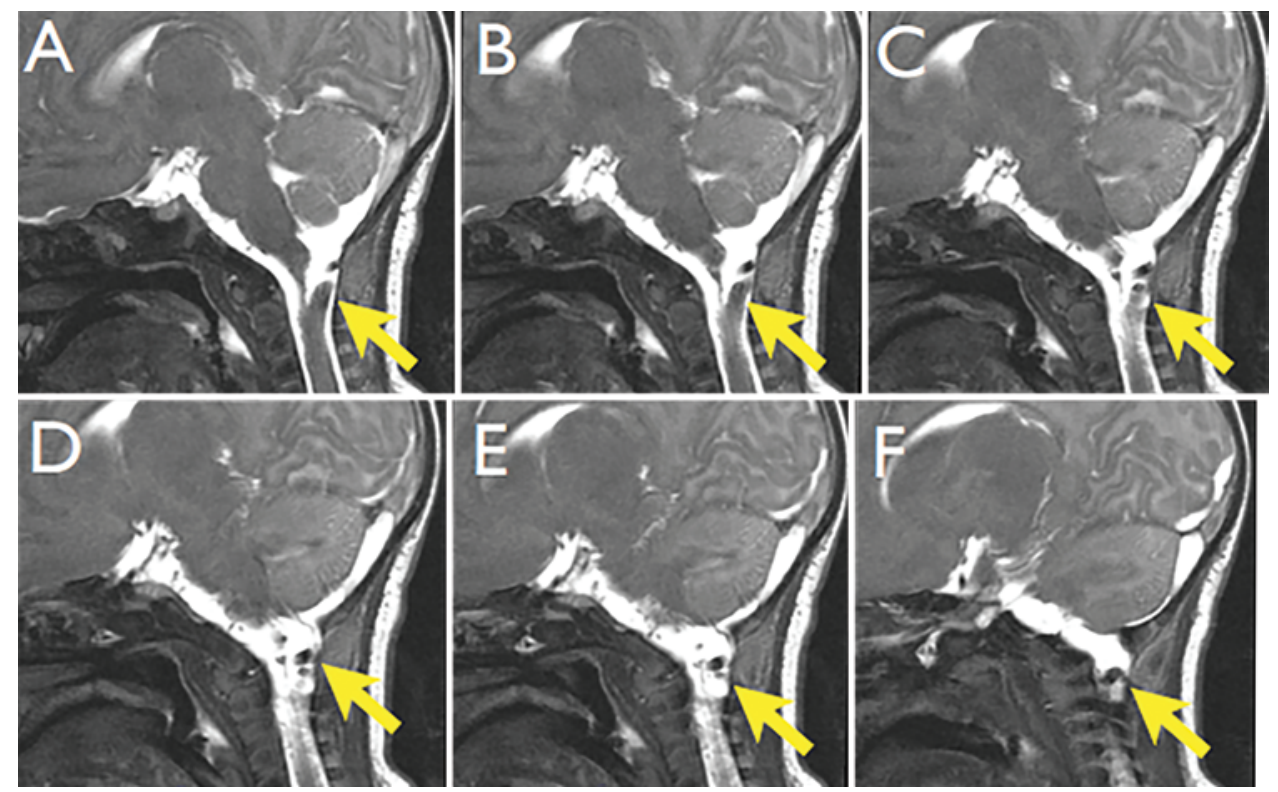

FIG. 2. Preoperative sagittal contrast-enhanced MR images showing the SCM (arrows). Figure is available in color online only. 


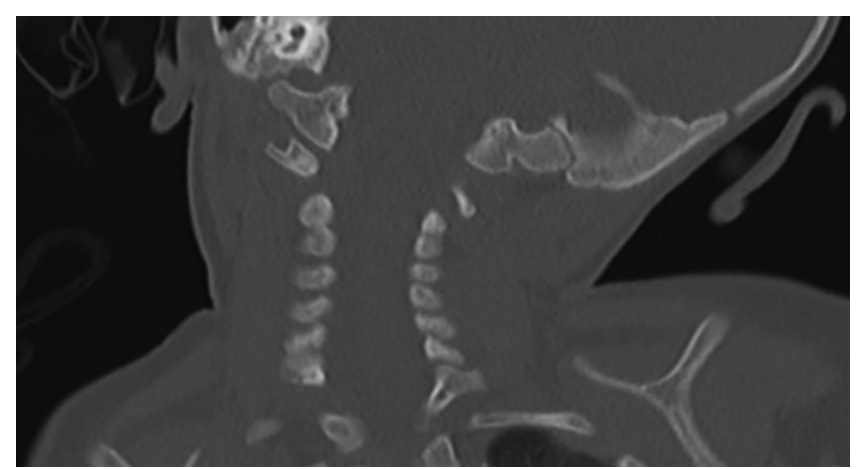

FIG. 3. Preoperative coronal CT scan showing left condylar and C-1 lateral mass hypoplasia.

\section{Operation}

The patient was taken to the operating room for a suboccipital craniectomy, cord untethering, and bovine duraplasty due to concerns for tethering of the upper cervical spinal cord and resultant compression contributing to cranial neuropathies and possibly central apnea and hypopnea. Large epidural varices were noted on the left and were coagulated with bipolar cautery. Upon dural opening, a large fibrous band was identified, appearing to split the cord (Fig. 4, Video 1).

VIDEO 1. Clip showing cervicomedullary junction exposure and excision of the fibrous band. Copyright Meghan E. Murphy.

Published with permission. Click here to view.

Adjoining the band was what appeared to be a separate aspect of the cervicomedullary junction and this was not surgically manipulated. The intradural band was excised at the dural margin. Once excised, the remaining edge of the band fell back between the hemicords of the cervicomedullary junction, completing the untethering. The left vertebral artery was identified deep to the fibrous band. Lower cranial nerves also appeared to be involved in the malformation. Finally, opening into the fourth ventricle was visualized without an arachnoid veil present. Generous bovine pericardium was used for watertight duraplasty. BAERs and electromyography from bilateral cranial nerves VII, X, XI, and XII were monitored. All

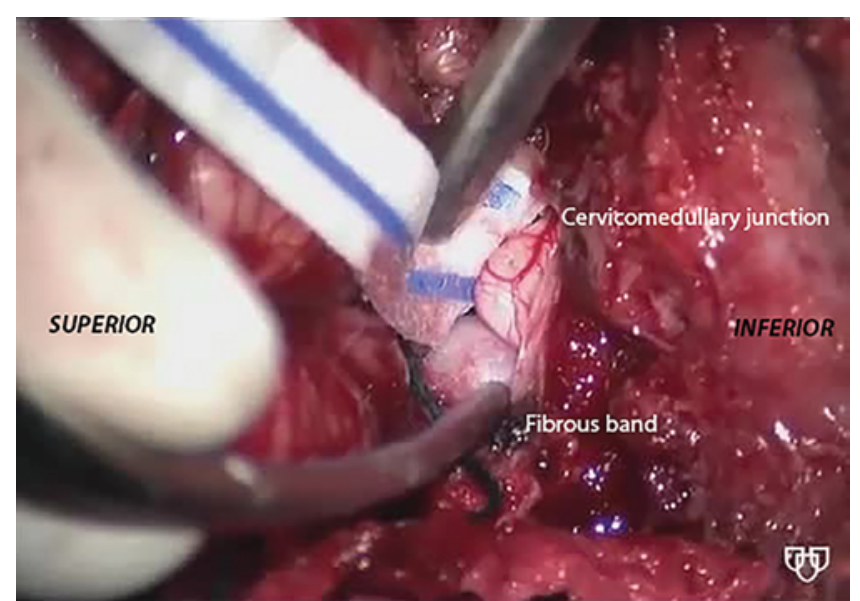

FIG. 4. Image captured from the intraoperative video. Figure is available in color online only.

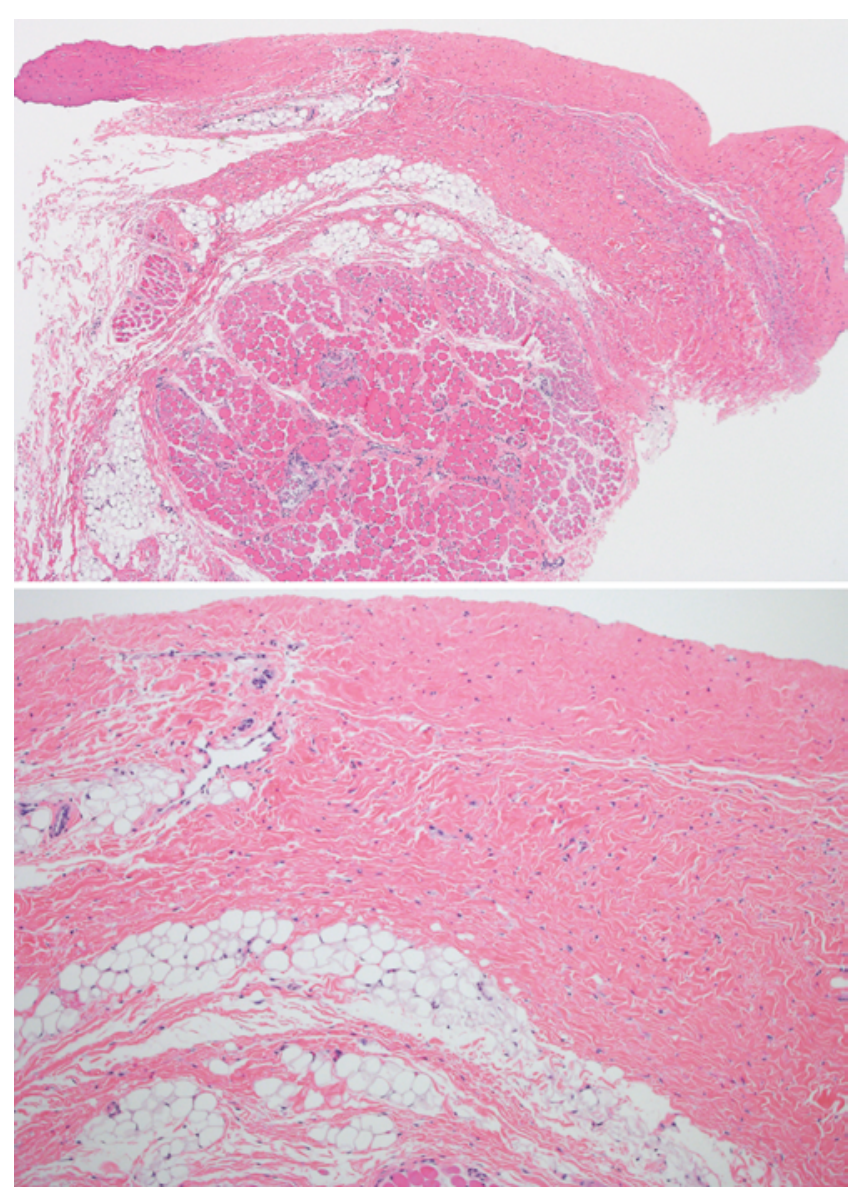

FIG. 5. Intraoperative frozen pathology specimen (upper, low power; lower, high power) showing skeletal muscle and fibrous tissue resembling fascia. Original magnification $\times 20$ (upper), $\times 40$ (lower). Figure is available in color online only.

waveforms of the BAERs remained stable throughout the procedure, and no neurotonic discharges were recorded. She tolerated the procedure well with minimal blood loss and was extubated. The immediate postoperative course was notable for respiratory distress requiring reintubation. She was successfully extubated on postoperative Day 1 and was transferred out of the pediatric intensive care unit on postoperative Day 2.

\section{Pathological Findings and Postoperative Course}

Pathological review of the specimen revealed skeletal muscle and fibrous tissue (Fig. 5). Postoperative imaging revealed decompression of the left skull base with resection of the intradural band. No cord signal abnormality or syrinx was observed (Fig. 6). She was discharged home on postoperative Day 4 with a supplemental oxygen regimen. On postoperative Day 5 she required readmission for respiratory distress and was treated with oral antibiotics for pneumonia. Bedside nasopharyngeal endoscopy revealed improvement of the left vocal cord mobility and overall good glottic closure; follow-up FEES revealed some evidence of penetration of thin liquids, but no frank aspiration, and she was subsequently continued on her oral diet. At 10 months of age, and nearly 3 months postopera- 


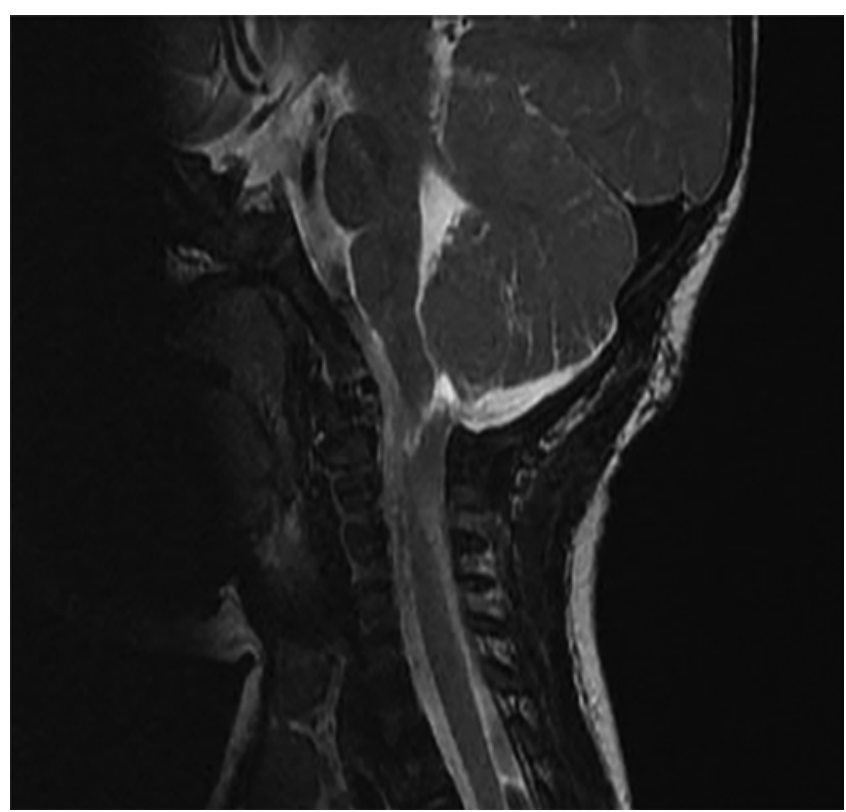

FIG. 6. Postoperative T2-weighted sagittal MR image showing resection of the fibrous intradural band and decompression.

tively, her torticollis was stable, and her parents noted decreased apneic events continuing on the nocturnal lowflow home oxygen therapy. At 2 years of age she demonstrated excellent developmental progression; she was crawling well and had approximately 50 words in her vocabulary. A polysomnogram performed on room air revealed a combination of obstructive and central apneas as well as hypopneas, with an AHI of 93. With the addition of low-flow oxygen at $0.25 \mathrm{~L}$ there was improvement in central apneas and hypopneas, but the obstructive sleep apneas were unaltered in frequency (the AHI was 39). She was subsequently referred for desensitization for positive airway pressure treatment. At last follow-up, 3 years of age and 29 months postoperatively, the patient continues to do well. She was tolerating positive airway pressure therapy overnight with minimal supplemental oxygen, room air while awake, and a general diet. She continues to make developmental gains, although she remains delayed in both cognitive processing and motor skills. As of her 4-year annual checkup, she is developing and growing normally. The torticollis remains stable without signs of any craniocervical instability.

\section{Discussion}

We hypothesize that the basic ontogenetic defect in our index case resulted in malformation of the lateral midbrain and formation of a short hemicord terminating in an aberrant segmental neural placode at the C1-2 level, with a normal single cord caudal to this lesion, thus suggesting an incomplete Type II cervical SCM. A Type II SCM, or diplomyelia, results in 2 hemicords separated by a fibrous septum contained within a common dural sheath. ${ }^{12}$

The hemicords grow caudally based on the development of their associated neural placode, found in all open and most closed spinal dysraphism cases. ${ }^{17}$ Importantly, these placodes can be defined either as terminal or seg- mental, depending on the final location. ${ }^{17}$ Caudal to a segmental placode, the spinal cord appears normal both morphologically and structurally. ${ }^{17}$ Patients may also have an associated meningocele manqué, which refers to the formation of tethering bands of fibrotic or atretic neural tissue connecting the spinal cord to dura or surrounding tissues, and may cause tethered cord syndrome (TCS). ${ }^{10}$ Because cervical SCMs are rare, their clinical impact is not well defined. Andro et al. presented a case of cervical SCM with associated vertebral anomalies, resulting in surprisingly mild symptoms. ${ }^{2}$ In contrast, our patient had a cervical SCM with profound neurological symptoms, including cranial neuropathies and unilateral motor deficits. This suggests that the anatomical location of the derangement drives both clinical impact and prognosis.

The unilateral vocal cord paresis and congenital torticollis noted in our patient likely resulted from involvement of the high cervical cord and ventrolateral caudal medulla. Vocal cord paralysis is the second most common cause of stridor in newborns, with an equal distribution between unilateral and bilateral vocal fold paralysis. ${ }^{4}$ Neurological etiologies represent $16 \%$ of reported cases; Chiari malformation is the most common. ${ }^{4}$ We hypothesize that the SCM and brainstem anomaly in our patient impacted the vagal nuclei, resulting in unilateral cord paresis. While there are several surgical interventions available for vocal cord paralysis, including tracheotomy, arytenoidectomy, and cord mobilization, our patient remained stable with conservative management and positive airway pressure therapy, and therefore did not require surgical intervention. The rapid improvement of swallowing and aspiration during the 1st month of her life was likely due to improved apnea of prematurity and her suck-swallow-breath reflex..11,14 The improved swallow study at Day 10 of life, in combination with her persistent cord weakness, supports this hypothesis. ${ }^{4}$

While rare, the most common etiology of congenital torticollis is birth trauma. ${ }^{8}$ However, neurological etiologies, including Chiari malformation, have been reported. ${ }^{1,5,13}$ This suggests that neurological injury to, or malformation of, the accessory motor nuclei or motor nerves within the accessory nerve itself may result in torticollis.

The diagnosis of closed spinal dysraphism and SCM is reliant in large part on careful physical examination and radiography. Most patients present with indicative cutaneous findings, yet patients can present without obvious clinical examination findings. ${ }^{6}$ In these situations, radiographic evaluation is the key to diagnosis. Diagnosis can be suggested with ultrasonography, plain radiographs, or CT.6,12 However, MRI is the ideal modality for evaluating SCMs. ${ }^{16}$ The key radiological findings in Type II SCMs are a single dural sac containing 2 hemicords, best seen on T2-weighted or FIESTA-weighted MRI, or the lack of a bone spur noted on T1-weighted imaging. In addition, evaluation of the thin fibrous septum may be possible with T2-weighted or FIESTA-weighted MRI. Notably, meningocele manqué is extremely difficult to diagnose on MRI alone, and often relies on surgical evaluation for definitive diagnosis. ${ }^{17}$ The tissue that appeared to be a separate component of the cervicomedullary junction intraoperatively may have been a meningocele manqué, atretic neural tis- 
sue tethered to normal elements. However, given the attachment to the primary cervicomedullary junction, this tissue was not manipulated surgically due to concern of damage to neural structures. The pathology of the excised intradural band showed mixed fibrous tissue consistent histologically with fascia.

Management depends largely on neurological sequelae, and is often dictated by the development of TCS. While TCS is classically believed to involve a tight filum terminale, it can be due to any condition that places tension on the spinal cord..$^{9}$ As in our case, the concern was tethering at the cervical level. Surgical intervention to release the tethered segment is often recommended; 6 however, surgery is not without risk, and therefore should be individualized to the patient and his or her risk of developing sequelae from the lesion. Signs of progressing neurological decline, such as pain or incontinence, should prompt the surgeon to consider release of the tethered segment. ${ }^{2}$

Long-term prognosis has been noted to be favorable in this patient population. ${ }^{15}$ Immediate postoperative concerns consist of general recovery from anesthesia and surgery, including respiratory status, tolerating a diet, pain control, and mobility. In addition, wounds are monitored closely for CSF leakage and infection. ${ }^{15}$ Patients are typically followed clinically and imaging performed as deemed necessary by their presentation and examination. Our patient underwent postoperative MRI and has been recommended to follow-up yearly, with special attention to symptoms of retethering or craniocervical instability.

This paper presents an exceptionally rare case of incomplete Type II cervical SCM and associated brainstem hypoplasia, resulting in multiple cranial neuropathies. The diagnosis relies on MRI and physical examination, and in the majority of cases conservative therapy with symptomatic management is sufficient. In our patient, conservative treatment was the first tier of clinical management. Due to worsening cranial neuropathies, progressive spinal cord tethering, and brainstem decompression, surgical intervention was merited. Her neurological status stabilized and she began to progress developmentally, albeit in a delayed fashion. The successful management of Type II SCMs relies on early diagnosis and close follow-up with a multidisciplinary team for the development of neurological sequelae. Early intervention can arrest progression of neurological injury.

\section{Acknowledgments}

Special thanks to Bruce A. Kall, MS, Assistant Professor of Neurosurgery, Mayo Clinic, Rochester, for his informational technology expertise in the formatting of the operative video.

\section{References}

1. Alexiou GA, Prodromou N: Torticollis as an initial sign of Chiari I malformation. Pediatr Emerg Care 25:215, 2009

2. Andro C, Pecquery R, De Vries P, Forlodou P, Fenoll B: Split cervical spinal cord malformation and vertebral dysgenesis. Orthop Traumatol Surg Res 95:547-550, 2009

3. Boix-Ochoa J, Lafuenta JM, Gil-Vernet JM: Twenty-four hour exophageal $\mathrm{pH}$ monitoring in gastroesophageal reflux. J Pediatr Surg 15:74-78, 1980
4. Daya H, Hosni A, Bejar-Solar I, Evans JN, Bailey CM: Pediatric vocal fold paralysis: a long-term retrospective study. Arch Otolaryngol Head Neck Surg 126:21-25, 2000

5. Dure LS, Percy AK, Cheek WR, Laurent JP: Chiari type I malformation in children. J Pediatr 115:573-576, 1989

6. Erşahin Y, Mutluer S, Kocaman S, Demirtaş E: Split spinal cord malformations in children. J Neurosurg 88:57-65, 1998

7. Galloway GM, Zamel K: Neurophysiologic intraoperative monitoring in pediatrics. Pediatr Neurol 44:161-170, 2011

8. Herman MJ: Torticollis in infants and children: common and unusual causes. Instr Course Lect 55:647-653, 2006

9. Hertzler DA II, DePowell JJ, Stevenson CB, Mangano FT: Tethered cord syndrome: a review of the literature from embryology to adult presentation. Neurosurg Focus 29(1):E1, 2010

10. Lassman LP, James CC: Meningocoele manqué. Childs Brain 3:1-11, 1977

11. Lau C, Smith EO, Schanler RJ: Coordination of suck-swallow and swallow respiration in preterm infants. Acta Paediatr 92:721-727, 2003

12. Pang D, Dias MS, Ahab-Barmada M: Split cord malformation: Part I: A unified theory of embryogenesis for double spinal cord malformations. Neurosurgery 31:451-480, 1992

13. Pellanda N, Mojon D: [Arnold-Chiari I malformation: three unusual manifestations.] Klin Monbl Augenheilkd 222:218-221, 2005 (Ger)

14. Poets CF: Apnea of prematurity: What can observational studies tell us about pathophysiology? Sleep Med 11:701707, 2010

15. Proctor MR, Scott RM: Long-term outcome for patients with split cord malformation. Neurosurg Focus 10(1):e5, 2001

16. Tortori-Donati P, Rossi A, Biancheri R, Cama A: Magnetic resonance imaging of spinal dysraphism. Top Magn Reson Imaging 12:375-409, 2001

17. Tortori-Donati P, Rossi A, Cama A: Spinal dysraphism: a review of neuroradiological features with embryological correlations and proposal for a new classification. Neuroradiology 42:471-491, 2000

\section{Disclosures}

The authors report no conflict of interest concerning the materials or methods used in this study or the findings specified in this paper.

\section{Author Contributions}

Conception and design: Wetjen, Maloney, Van Abel. Acquisition of data: Wetjen, Maloney, Murphy, Sullan, Van Abel. Drafting the article: Maloney, Murphy, Sullan, Van Abel. Critically revising the article: Wetjen, Maloney, Murphy, Van Abel, Cofer. Reviewed submitted version of manuscript: Wetjen, Maloney, Murphy, Van Abel, Cofer. Administrative/technical/material support: Murphy, Cheville. Study supervision: Wetjen, Cofer. Pathology slides: Cheville.

\section{Supplemental Information \\ Videos}

Video 1. https://vimeo.com/187187690.

\section{Correspondence}

Nicholas M. Wetjen, Department of Neurologic Surgery, Mayo Clinic, 200 First St. SW, Rochester, MN 55905. email: wetjen. nicholas@mayo.edu. 